\title{
The Role of Viruses and Viral Infections in the Theory of Panspermia
}

Koushik Chattopadhyay*

F. Widjaja Foundation Inflammatory Bowel and Immunobiology Research Institute, Department of Gastroenterology, Cedars-Sinai Medical Center, Los Angeles, California, USA

*Corresponding author: Dr. Koushik Chattopadhyay, F. Widjaja Foundation Inflammatory Bowel and Immunobiology Research Institute, Department of Gastroenterology, Cedars-Sinai Medical Center, Los Angeles, California, USA, Tel: 724-503-5855; E-mail: chatt_k@yahoo.com

Rec Date: March 1 2014; Acc Date: May 29 2014; Pub Date: May 312014

Copyright: ( 2014 Chattopadhyay K. This is an open-access article distributed under the terms of the Creative Commons Attribution License, which permits unrestricted use, distribution, and reproduction in any medium, provided the original author and source are credited.

\begin{abstract}
Panspermia is an ancient idea that supports the existence of life throughout the Universe. Panspermia proposes that life that can survive the effects of space get distributed in all habitable and non-habitable planets by meteoroids, asteroids and planetoids across the Universe. Extremophilic micro-organisms known to survive and thrive in extreme conditions on earth also pose a possibility of existing in other parts of universe delivered by comets or asteroids. Viruses, being simple organisms, are also capable of such survival and journeys across space. Viruses are known to be mobile genetic elements and install new genes into their host cells supporting the evolutionary mechanism. This article illustrates the role of viruses and viral infections in the theory of panspermia by examples of horizontal gene transfer, influenza virus and SARS.
\end{abstract}

Keywords: Virus; Panspermia; Origin of life; Extremophilic

\section{Introduction}

The origin of life on earth has for long been debated. This is probably one of the biggest puzzles in biology and science which is yet to be fully understood. Panspermia is a highly controversial theory that proposes the theory of origin of life on earth hypothesizing the arrival of life on earth from outer space. Extremophilic microorganisms capable of surviving in extreme conditions have been proposed to travel far into space unaffected by means of cometary materials and subsequently been proposed to flourish in a distant planet that harbours favourable conditions. Viruses which are a simple form of life are hypothesized to be potential candidates for space travel. The theory of panspermia have been supported by examples of different viruses, their predicted origin and spread on the earth as well as ability to carry out horizontal gene transfer to answer questions of origin of life on earth and its subsequent evolution to higher forms. This article will attempt to address the understandings of the theory of panspermia and the roles of viruses in supporting this theory.

\section{Origin of Life on Earth}

Different theories have been proposed to explain the origin of life on earth. However, the most widely regarded is that of life originating from a primordial soup in primeval earth. This theory originally proposed by Oparin [1] and Haldane [2] and supported by experiments of Miller [3] explains that the primordial atmosphere on the earth facilitated the formation of first organic molecules. Atmospheric organic and inorganic matter such as hydrogen, carbon, methane, ammonia, nitrogen through the action of solar ultraviolet radiation and electrical discharges synthesize organic molecules or amino acids facilitated by the primordial atmosphere on earth. Amino acids are the chemical building blocks of life. These organic molecules got dispersed into the primitive oceans via rain which resulted in a primordial soup. However, this soup, too dilute to support complex biochemical reactions for transformations of the organic molecules to any form of life, eventually transformed into a concentrated primordial soup through millions of years of evaporation of water under extreme heat conditions on earth. Further chemical transformations on the primordial soup possibly generated the first self-replicating living cells, the first form of life on earth. The formation of organic compounds from inorganic molecules is supported by experiments [4]. However the further steps leading to origin of life have not been experimentally proven thus far.

Our current understanding does not provide any knowledge that the final step of formation of life from organic molecules took place on earth. The discovery of the amino acid glycine in Stardust-returned foil samples [5] strongly suggests an extraterrestrial origin for glycine potentially delivered by comets that survived the conditions in early Earth. The possibility of glycine reaching its end state in comets by forming a molecular bond with some species of cometary dust or amorphous ice has also been shown experimentally [6,7]. A recent study using computer models also showed that the complex organic molecules that are the building blocks for life on Earth may have formed in the protoplanetary disk of dusty grains that surrounded the Sun before the formation of the planets [8]. These lead us to the justification of considering other alternative theories that consider the entire Universe as a platform for origin of life. The final information facilitating the transformation of life from non-life compounds could have arrived on earth which is constantly exposed on our vast cosmic system.

\section{Panspermia}

The term Panspermia is a Greek word that literally means "all seeds". This theory proposes that life exists throughout the Universe and is widely distributed in the form of germs or spores [9]. Panspermia does not address the origin of life per se but the method that may have caused its sustenance [10]. According to the theory of panspermia, life such as extremophilic archaea that can survive the effects of space get trapped in the debris of cometary materials such as meteoroids, asteroids and planetoids [9]. These micro-organisms remain dormant in this debris until they come in contact with ideal 
conditions on a new planet's surfaces when these micro-organisms become active and start replicating [10].

The hypothesis of panspermia is an ancient theory. Vedic philosophy, one of the most ancient, considers life as eternal and an inseparable part of the Universe. A 5th century BC philosopher Anaxagoras first mentioned the term of panspermia in his writings [11]. The idea of panspermia was revived in nineteenth and twentieth century by several scientists like Hermann von Helmholtz of Germany and Svante Arrhenius of Sweden [12]. Sir Fred Hoyle and Chandra Wickramasinghe are two of the strongest proponents of this hypothesis in modern times who support that life on earth is derived from a vast cosmic system [13-15]. They further argue that life forms and genetic information constantly enter the Earth's atmosphere from space and might be responsible for evolution on earth, epidemic outbreaks and new diseases [16].

\section{Extremophilic Microorganisms}

The microorganisms that survive and thrive in extreme environments like high pressure, high heat, below freezing temperatures, high alkalinity or lack of oxygen are termed as extremophilic microorganisims. These microorganisms could also potentially survive in planets other than earth with similar extreme conditions or in comets or meteoroids. If microorganisms are sheltered inside a comet or a meteoroid and escape the harsh radiation of space, they can remain dormant for millions of years and become active after being transferred to a planet with favourable conditions. Experiments conducted by the Martian Simulation Laboratory (MSL) have reported remarkable adaptation capacity of lichens with regards to their survival and photosynthetic ability in Martian conditions [17].

Geological evidence suggests that the first life on Earth appeared in the form of algae around 3.83 billion years ago, at a time that is well known for heavy bombardments of comets and meteorites on Earth [18]. It is possible that some types of microbial life that were adapted to high pressure and subsurface conditions could have been trapped in cometary materials and transferred to Earth during this period [15].

\section{Virus and Their Roles in Panspermia}

A cell is an independent living entity that can eat, grow and reproduce. Unicellular bacteria are not much different from human or mammalian cells with regards to their complex cellular make up and processes though much smaller in size. A virus, on the other hand, is a simpler form of life and resembles no similarity to living cells. Viruses are not classified as typical living cells. Rather a virus is a piece of genetic information (DNA or RNA) within a protective coat. They lack all the machinery a cell possesses to survive and replicate, other than a small quantity of DNA or RNA. Viruses transform into living particles only when inside their particular host cells being able to metabolize and reproduce. Once outside their host cells, they are just a non-living complex molecular particle that have no metabolic activities and no way to reproduce. Due to these characteristics viruses were thought to be the pre-cellular life forms that link non-living materials with living beings. Though this notion has fallen apart, the viruses are still believed as one of the most ancient forms of life. Around 4,000 different types of viruses have been characterized which is just a tiny fraction of the total number of types of viruses present on Earth. Having no independent metabolism and reproductive machinery, they can remain viable in a dormant state outside their host cells for indefinite periods of time under the right circumstances.
Some of the viruses can even be crystallized like minerals in their nonliving state. In this state the viruses can survive for millions of years unchanged until they are come in contact with moisture and their particular hosts. Inside the right host cell and with favourable conditions, the dormant viruses can become active and begin to metabolize and reproduce $[19,20]$. These characteristics of a virus makes it a perfect candidate for travel through space inside the protective shields of comets or meteoroids. As it is well known that amorphous ice is abundant in the depths of interstellar medium [21], the virus might also get trapped in the amorphous ice inside comets or asteroids and spread life in a planet that offers a host with a favourable condition for life to survive and replicate.

\section{Horizontal Gene Transfer}

Transfer of genetic material between different organisms across species is known as horizontal gene transfer. This is a strong tool that aids in the evolutionary mechanism. Viruses are mobile genetic elements that spread genes among bacteria and different living species including humans [22]. Once inside a host cell, the virus causes the host cell to go through either lytic or lysogenic cycle. In the lytic cycle, the host cell replicates the virus and when several copies are made, the cell breaks open and dies and the many copies of viruses are released. In the lysogenic cycle, a virus is silently incorporated into a host cell's genome. The host cell keeps performing like a normal cell. In this phase the virus is latent and waits for the right opportunity and favourable conditions to get into the lytic cycle to start replicating and spreading again. A virus takes up a piece of DNA from its host cell and incorporates it into its own viral genome (or vice versa) through the process of transduction. The genetically modified virus then gets incorporated into another host cell's genome and the process of shuffling genetic material continues $[23,24]$. Large blocks of genetic information can thus be swapped and transferred among different organisms. The horizontal gene transfer by viruses through insertion and shuffling of genes across species boundaries could be the main driving force behind the evolutionary mechanism.

By this process of merging with the cellular genome and reemerging from them, the virus shuffles lots of genetic information across species and could easily provide a way for new genes never before encountered by a species to be a part of its genome. Thus, viruses play a strong role in genetic evolution of all eukaryotes including human beings and are probably still contributing. Evidence for this can be found in the enormous presence of cryptic, incomplete, retroviral sequences in human genome [25]. Shuffling of genes can also trigger mutations, paving way for more changes to the genetic information. By mechanisms mentioned above, the viruses create an ever changing gene pool in the kingdom of life which, in the course of evolution, has created the successful diverse genetic patterns that we see in today's world.

\section{Viruses from Space}

It has been speculated by scientists that around 1,500 years ago when a comet hit the Earth, it had spread some kind of a plague which was believed to be responsible for the collapse of the Roman civilization into dark ages. However no scientific proof has been found in support of this theory. 


\section{Influenza Virus}

Influenza which is commonly known as the flu, is an infectious disease caused by RNA viruses of the family Orthomyxoviridae. Influenza makes a strong case of evidence in favour of panspermia. It is claimed that influenza outbreaks were caused by newly arriving viruses from space. The influenza outbreak of 1918-19 took several days and weeks to travel to short distances, but appeared on the same day in widely separated parts of the world. The outbreak is believed to have started in Boston (USA) and in Mumbai (India) at the same time; in 2 sites situated in 2 different continents. But interestingly, it took almost three weeks to travel 190 miles from Boston to New York City (USA). The pandemic also started suddenly during winter in 1918 in villages in Alaska which are well isolated from rest of the world during winter. Interestingly, it has been noticed that the worst flu epidemics coincide with peaks in the eleven-year cycle of sunspot activity. This was confirmed by an unusually vigorous flu epidemic that matched the pattern in January, 2000 [26].

A subtype of influenza virus was only detected in Sardinia Island in 1948 that was quite isolated at that time. Influenza was also detected in 1949 in shepherds who were living alone for a long time, in solitary open country far from any inhabited centre. Van Loghem monitored 7000 people in Netherlands from September-June in 1925-26 for occurrence of cold. He noticed that the time of rise and fall of the cold was exactly same in different places and also the extent of the rise was similar [27]. Respiratory infections due to cold are always reported from middle to late winter irrespective of the hemispheres. They occur six months apart in northern and southern hemispheres according to the start of the winter in the respective hemispheres. This suggests a major atmospheric effect on the occurrence of flu virus and also a relation of the earth's axis of rotation to the direction of the Sun. In 1958 an atmospheric tracer experiment was conducted with rhodium (Rh)-102 [28]. Rh-102 was a special tracer created by neutron activation in a rocket-borne nuclear device detonated $43 \mathrm{~km}$ above ground level at a remote island in Pacific Ocean. The resulting nuclear debris cloud rose far above the stratosphere, roughly $100 \mathrm{~km}$ and spread across the world. Later on Rh-102 was collected $20 \mathrm{~km}$ above ground level suggesting it was able to penetrate through the atmosphere. The concentration of Rh-102 was highest during JanuaryFebruary [28]. It suggests that if indeed the flu virus comes from the space, it is also able to penetrate through the atmosphere and expected to rise in number during winter months, which is what was observed in reality. It has been hypothesized by the believers of panspermia that the closely related viral sequences are introduced from space and have a residence time of a decade. They remain suspended in the upper atmosphere, particularly in the stratosphere, that creates a reservoir of viruses. They are pulled down the atmosphere seasonally and circulated throughout the globe by atmospheric circulation. It is likely that one season only supports the circulation of one virus. Since the human immune system is evolving with exposure to new strains of influenza virus, earlier subtypes are replaced by genetically altered stronger viruses in subsequent seasons.

\section{SARS}

Panspermia theorists have suggested a strong likelihood of an extraterrestrial origin for the SARS virus [29]. SARS is a type of coronavirus but bears unexpectedly novel genetic sequences compared to other coronaviruses. This points towards a possibility that the virus might have evolved independently from outside the earth. The virus appeared without warning in mainland China. It is plausible that a small amount of the virus could have fallen out from the space onto the East of the Himalayas where the stratosphere is thinnest, followed by sporadic deposits in the surrounding areas. Since the virus is minimally infective, its global progress depends on stratospheric transport and mixing. Seasonal fall out of the virus is expected to continue until the supply becomes exhausted. Meanwhile, new strains of the virus might continue to appear in places across the globe [29].

\section{Counter Argument}

There had been criticism to the idea of panspermia in regards to viruses and their origin. RNA viruses survive only hours to days and are hard to explain their fallout from the space and still remain active. Viruses are completely dependent on host cells for their survival and metabolic activities which question their independent, extraterrestrial origin. Evolutionary analysis of the protein-coding sequences of the SARS virus has shown similarities with mammalian and avian coronaviruses already known. No new strains of SARS have emerged until now unlike as predicted by the panspermia theorists.

\section{Conclusion}

It is evident that the theory of panspermia has obvious reasons to be controversial. It is an alternative theory that considers the earth and the life forms as a part of the bigger Universe and the whole Universe as a grand crucible of cryobiology. The origin of viruses, virus related infections and their spread have traditionally been used by the panspermia theorists to support and explain their hypothesis. The counter arguments pose strong logic as well, although the proponents of the panspermia theory are growing quite rapidly. The purpose of this article is not to support or refute the hypothesis or its explanations but to create an understanding in the minds of readers for them to assess the merits and demerits of this theory as per their own judgement. Viruses have posed great challenges to scientists since their discovery about their existence and scientists across the globe have addressed these challenges through different hypotheses. Till date viruses and virus related infections remain a puzzle for us and we continue to seek explanations for many problems hitherto unknown.

\section{Acknowledgement}

"I would like to thank Dr. Uthra Rajamani for helping to format the manuscript and also correct the grammatical errors."

\section{References}

1. Oparin AI (1953) The origin of life. Dover Publications, New York.

2. Haldane JBS (1929) The origin of life. Chatto and Windus

3. Miller SL (1953) A production of amino acids under possible primitive earth conditions. Science 117:528-529

4. Parker ET, Cleaves HJ, Dworkin JP, Glavin DP, Callahan M, Aubrey A, Lazcano A, Bada JL (2011) Primordial synthesis of amines and amino acids in a 1958 Miller H2S-rich spark discharge experiment.

5. Elsila JE, Glavin DP and Dworkin JP (2009) Cometary glycine detected in samples returned by Stardust. Meteorit Planet Sci 44: 1323-1330.

6. Escamilla-Roa E, Moreno F (2012) Adsorption of glycine by cometary dust: Astrobiological implications. Planet Space Sci 70: 1-9.

7. Escamilla-Roa E and Moreno F (2013) Adsorption of glycine by cometary dust: Astrobiological implications. Planet Space Sci 75: 1-10.

8. Moskowitz C (2012) Life's Building Blocks May Have Formed in Dust Around Young Sun. 
Citation: Chattopadhyay K (2014) The Role of Viruses and Viral Infections in the Theory of Panspermia. Astrobiol Outreach 2: 111. doi:

Page 4 of 4

9. Rampelotto PH (2010) Panspermia: a promising field of research. In: Astrobiology Science Conference.

10. Grossman L (2010) All life on earth could have come from alien zombies. Wired Science

11. O'Leary M (2008) Anaxagoras and the origin of panspermia theory.

12. Arrhenius S (1908) Worlds in the making: The evolution of the Universe. Harper and Row, New York.

13. Hoyle F, Wickramasinghe NC (1999) Astronomical origins of life: steps towards panspermia. Kluwer Academic Publisers, Dordrecht.

14. Hoyle F, Wickramasinghe NC, in Ponnamperuma CE (1981) Comets and the origin of life. Reidel, Dordrecht: 227

15. Wickramasinghe C (2004) The universe: a cryogenic habitat for microbial life.

16. Hoyle F, Wickramasinghe C, Watson J (1986) Viruses from space and related matters. University College Cardiff Press, UK

17. de Vera JP, Kohler U (2012) The adaptation potential of extremophiles to Martian surface conditions and its implication for the habiyability of Mars. European Geosciences Union General Assembly.

18. Mojzsis SJ, Arrhenius G, McKeegan KD, Harrison TM, Nutman AP Friend CR (1996) Evidence for life on Earth before 3,800 million years ago. Nature 384: 55-59.

19. Waldor MK, Mekalanos JJ (1996) Lysogenic conversion by a filamentous phage encoding cholera toxin. Science 272: 1910-1914.
20. Williams N (1996) Phage transfer: a new player turns up in cholera infection. Science 272: 1869-1870.

21. Debenedetti PG, Stanley HE (2003) Supercooled and glassy water. J Phys: Condens. Matter 15: 45.

22. Alberts B, Bray D, Lewis J, Raff M, Roberts K, Watson JD (1994) The molecular biology of the cell. Garland Publishing, New York.

23. Baserga SJ, Steitz JA (1993) The diverse world of small ribonucleoproteins. Cold Spring Harbor Laboratory Press: 359-381

24. Gesteland RF, Atkins JFe (1993) The RNA world. Cold Spring Harbor Laboratory Press:374

25. Luria SE (1959) Virus growth and vaccination. Cambridge University Press: 110

26. Hoyle F, Wickramasinghe C (2000) The dilemma of influenza. Current Science (from the Indian Academy of Sciences).

27. Van Loghem JJ (1928) An Epidemiological Contribution to the Knowledge of the Respiratory Diseases. J Hyg (Lond) 28: 33-54.

28. Telegadads K, List RJ (1964) Global history of the 1958 nuclear debris and its meteorological implications. J Geophys Res 69: 4741-4753.

29. Wickramasinghe C, Wainwright M, Narlikar J (2003) SARS--a clue to its origins? Lancet 361: 1832 